\title{
What scales the $T$ cell response?
}

$4 \quad$ Viveka Mayya $^{1}$ and Michael L. Dustin ${ }^{1,2, *}$

5

$6{ }^{1}$ Kennedy Institute of Rheumatology, University of Oxford, Roosevelt Drive, Headington, 7 Oxford, OX3 7FY, UK.

$8{ }^{2}$ Skirball Institute of Biomolecular Medicine, NYU Medical Center, New York, NY 10016, 9 USA

$10 \quad{ }^{*}$ Correspondence: michael.dustin@kennedy.ox.ac.uk; +44 (0)1865 612639 (Ph); +44 (0)1865 11612601 (Fax)

\section{Abstract}

15 T cells are known to scale their clonal expansion and effector cytokine response according to the 16 dose and strength of antigenic signal in order to balance their role of affecting protection with the 17 intertwined, immunologically driven tissue damage. How T cells achieve this is now beginning 18 to be understood. We underscore temporal integration of digital TCR signaling as the basis for 19 achieving scaled response by means of accumulating critical mediators over time. We also 20 discuss the role of temporally integrated cross-talk between TCR and IL2 signaling, in mediating 21 a scaled, coherent, collective response by T cells. Finally, we highlight numerous known and 22 putative regulatory interactions in the transcriptional program that are expected to quantitatively 23 scale the $\mathrm{T}$ cell response and also offer new mechanisms to hitherto unexplained observations. 


\section{Scaling of the $\mathbf{T}$ cell response}

2 An optimal immune response is critical to nullify the pathogenic threat while ensuring that the

3 host is not irreversibly disadvantaged by the various effector mechanisms. Innate cells evaluate

4 the level and type of microbial threat for a scaled anti-microbial response [1]. This is translated

5 to the adaptive arm of the immune system by the dendritic cells. Naïve T cells for any antigen

6 are present in very low frequency and need to undergo clonal expansion by 10-20 rounds of cell

7 division upon activation by dendritic cells [2, 3]. T cell expansion scales with respect to the

8 initial inoculum of the pathogen and the level of expression of the antigen [4-6]. Similarly

9 cytokine expression scales with the dose of the antigen ex vivo and in vivo [5, 7]. Such scaling is also observed in response to infection by a panel of recombinant $L$. monocytogenes that give rise to altered peptide ligands (APL) representing a wide range of TCR-MHCp affinity and in response to direct APL immunization [5, 8-10]. It must be noted that the scaling can plateau at very high antigenic dose or strength, but the collective evidence points to scaling occurring over a wide range $[5,6]$. A sub-optimal T cell response, be it in clonal expansion or in effector molecule expression, can result in failed or delayed clearance, chronic infection or escape of a tumor [6], for example. On the other hand, an over-blown response, especially clonal expansion, is wasteful and is expected to put strain on the resources of an already distressed host. More importantly, an overt T cell response can cause excessive tissue damage and potentially exacerbate or initiate autoimmune reactions $[9,11]$. These observations argue for scaled response being an important functional feature of the immune system.

\section{Temporal integration for scaling the $\mathbf{T}$ cell response}

23 Recognition of a cognate MHCp by the TCR leads to a signaling cascade followed by a transcriptional cascade resulting in the expression of cytokines, and master regulators of cell cycle, anabolic processes, and differentiation [12,13]. Examination of activation status of

26 multiple enzymes and transcription factors in stimulated cell populations has revealed that the

27 TCR signaling system functions as a digital switch with very limited dynamic range in their

28 levels among the cells activated with varying doses of antigen (Supplementary Note 1) [14]. Ex

29 vivo investigations have shown that escalating fraction of cells respond with increasing dose or 
affinity of antigen (Supplementary Note 1) [14]. Thus, varying the fraction of cells recruited to the response can theoretically give up to 100-fold dynamic range. However, in response to an infection, essentially all the cognate $\mathrm{T}$ cells are recruited irrespective of the pathogen, antigen dose and affinities for the MHCp $[4,6,8]$. This near complete recruitment is thought to breakdown only if affinity comes close to that of self-peptides or if the subdominant epitope is expressed or presented at very low levels $[6,8,15]$. Then how do $\mathrm{T}$ cells achieve scaling of their response according to the dose and strength of the antigens they encounter?

There is a large body of evidence to conclude that the $\mathrm{T}$ cell activation process is reliant on temporal integration of signaling [14]. This can be classified into four groups: 1) Firstly, the prerequisite for temporal integration, i.e. continuous or prolonged signaling, is satisfied during $\mathrm{T}$ cell activation [16]. Although there is ligand-induced degradation of TCR, de novo synthesis and receptor recycling ensures that a lower steady state level of surface TCR is maintained during continuous signaling [16-18]. Since this steady state level of TCR is still in vast excess compared to cognate MHCp, time-integrated TCR-MHCp is expected to scale linearly with MHCp [14, 16, 19]. 2) T cells have been shown to integrate from discontinuous signals due to hysteresis and stability of signaling intermediates and transcription factors [20-23]. 3) The commitment to proliferation or production of cytokines is delayed when $\mathrm{T}$ cells are presented weaker signals [5] by means of lower dose [16] or affinity of antigen [24] or withdrawal of co-stimulation [16]. 4) Finally, the extent of proliferation scales with the experimentally manipulated duration of antigenic signaling in vivo, which has been demonstrated by multiple groups by multiple approaches [3]. This forms the most crucial set of evidence from the standpoint of scaling. But, how does temporal integration lead to scaling?

\section{Scaled accumulation of critical mediators by temporal integration}

From a mechanistic viewpoint, transcription and translation are inherently time-integrated processes as the products can continue to accumulate over prolonged periods of signaling. When prolonged or continuous signaling is coupled to transcription, small quantitative differences in the signaling intermediates, as would be the case for the TCR signaling cascade, can faithfully be read into the build-up of translated products over time. This should provide convenient means to 
1 a large dynamic range and a linear response, an assertion best exemplified by studies from Dario

2 Vignali's group on manipulating the multiplicity of ITAMs in the CD3 complex. The extent of

3 proliferation, as measured by thymidine incorporation, was linear with respect to the number of

4 ITAMs in the CD3 complex, despite no significant difference in immediate cytokine production

5 [25]. Proliferation scaling in relation to ITAMs was later shown to be due to marginal increase in

6 Vav1 association with CD3 resulting in increased Notch1 activation and subsequent scaling in

7 Myc transcription, while the activation status of other signaling intermediates such as Zap70 and

8 Erk were indistinguishable [26]. Myc leads to global transcriptional amplification and also drives

9 essentially the entire biosynthetic and cell cycle machinery [27-29]. Similarly, notch1 and notch2 together contribute to nearly half of the gene expression changes in activated $\mathrm{T}$ cells due to their ability to target the biosynthetic and cell cycle machinery like Myc [30, 31].

There is a second set of evidence supporting the accumulation of critical mediators of the T cell responses due to temporal integration. Continuous TCR signaling for 24-48 hours is sufficient to program the cells to then undergo a set number of divisions in an antigen-independent manner, but as per the strength of the initial antigenic signal $[3,10]$. Inflammatory and co-stimulatory signals are additive in this early period of signaling, which further increase the number of divisions the cells undergo later [10]. This is possible if critical regulators of the biosynthetic and mitotic machinery accumulate proportional to the sum of all three signals and then get diluted during each round of division due to lack of any additional accumulation. This assertion is also supported by the observation that time taken for later divisions increases [32]. Two recently described transcription factors necessary for CD8 T cell clonal expansion and effector differentiation, BATF and IRF4, have the suitable characteristics to enable scaled response in the context described above: 1) Gradual accumulation of IRF4 over the first 24-48 hours scales according to antigenic dose and strength ex vivo [33, 34]. 2) IRF4 drives majority of the affinitydependent gene expression, which includes hundreds of metabolic and biosynthetic enzymes [33]. 3) Haploid status for IRF4 and BATF results in concordant reduction in clonal expansion and expression of immune effector molecules in vivo [33, 35]. 4) Knock-down of BATF 72 hours after LCMV infection by IPTG-inducible shRNA expression does not impact the T cell response [36]. This would mean that BATF attains the maximal required accumulation within $\sim 60$ hours. 5) IRF4 and BATF are not required to initiate the first $\sim 4$ divisions [33, 35, 37]. 
However they are important for sustained clonal expansion by means of driving cellular growth,

2 repressing CDK inhibitors and preventing apoptosis.

3 Amplification arising out of temporal integration can convert heterogeneity in expression of

4 signaling proteins into a large diversity in functional outcome in individual cells [38]. Indeed,

5 recent data from single-cell-centric experiments have revealed a diversity of individual and

6 clonal behavior in T cells responding to infection [39]. How do we then observe consistent

7 responses despite the large diversification? Experimental and theoretical analyses independently

8 reported by three laboratories have shown that population averaging of fates from as few as 50

9 naïve cells is sufficient to obtain consistent responses between mice [39]. Theoretical analysis of

10 fates of B cells that were activated ex vivo pointed out that stochastic choice between competing,

11 mutually exclusive outcomes in a cell autonomous manner is sufficient for reproducible,

12 averaged responses in a small population of cells [40]. Nonetheless, T cell expansion and

13 differentiation does not occur in a fully autonomous manner and is known to be plastic and

14 shaped by several cytokines [2, 3, 41, 42]. This suggests that responding T cells attempt to

15 reduce divergent responses by means of paracrine effects of cytokines and do not rely solely on

16 the statistical action of the central limit theorem [43]. Recent studies have shed light onto how

17 the interplay between cytokine and TCR signaling facilitated by temporal integration leads to

18 scaled, coherent, collective responses.

\section{Cross-talk with IL2 signaling for collective scaling}

21

22

23

24

25

26

27

28
Interleukin-2 (IL2) is a cytokine that is critical for sustained clonal expansion of responding T cells [2]. Scaled production and secretion of IL2 is therefore likely to be important to drive scaled expansion. IL2 signaling is known to repress further transcription of Il2 locus, which would set a ceiling on the extracellular accumulation of IL2 [44, 45]. Thus it remained an enigma as to how scaled production of IL2 and subsequent expansion is achieved. Altan-Bonnet and colleagues have addressed this enigma in their recent study by finding that TCR signaling inhibits Stat5 phosphorylation (Figure 1a) [7]. Tyrosine phosphorylation of some of the Stat transcription factors is prevented by serine phosphorylation and subsequent sumoylation at adjacent residues, which may be enforced on Stat5 by TCR signaling [46, 47]. Regardless of the 
mechanism, inhibition of Stat5 acts against rapid and robust repression of transcription of the Il2

2 locus by IL2 signaling. Thus, IL2 continues to accumulate for $\sim 48$ hours of continuous TCR

signaling (Figure 1b). Altan-Bonnet and colleagues have identified two additional features that enhance the scaling in time-integrated, collective production of IL2 over the first $\sim 48$ hours: 1)

5 Gradual increase in the number of cells secreting IL2. 2) Non-linear increase in rate (i.e. acceleration) of secretion of IL2 on a per cell basis [7] (Figure 1b). These ultimately enable over 3 orders of magnitude scaling in IL2 accumulation within $\sim 48$ hours in response to 4 orders of

8 magnitude difference in antigen dose, irrespective of 2 orders of magnitude variations in the starting number of antigen-specific T cells [7]. The authors have postulated the presence of an intermediate step with a positive feedback for driving the accelerated accumulation of IL2 in response to continuous TCR signaling (Figure 1a).

Beyond the first $\sim 48$ hours, time-integrated accumulation of a critical amount of IL2 receptor (IL2R) due to TCR signaling kick-starts the positive feedback of further transcription of IL2R subunits due to IL2 signaling (Figure 1a) [44]. This positive feedback is mediated directly by

Stat5 and indirectly by Notch expression and activation [31, 48]. Accumulated IL2R quickly begins to consume available IL2 due to internalization and degradation of the IL2R-IL2 complex. Further, dominant role of IL2 signaling will also suppress transcription of the Il2 locus leading to gradual depletion of IL2 (Figure 1a, 1b). This regulatory network enables accumulated IL2 to drive a concordant number of divisions after 24-48 hours, which can proceed in an antigen-independent manner [3, 44]. However, the presence of antigen beyond $\sim 48$ hours is expected to prolong the availability of IL2 and IL2R and thus drive additional expansion, as observed in vivo [3].

IL2 is known to have paracrine effects with an effective range of few tens of $\mu \mathrm{m}$, which will impact the immediate surrounding cells [45]. Paracrine messengers secreted from cells that produce them in larger quantities can either trigger secretion of a secondary messenger from nonproducers and/or trigger responses in all the cells in vicinity due to diffusive spread [49, 50]. Thus, a paracrine messenger can be a shared messenger for a collective, coherent response. IL2 has also been shown to be a shared messenger: 1) primed T cells form homotypic aggregates with IL2 production and consumption occurring in a polarized fashion in these aggregates [51].

2) Cells responding to a weaker antigen can co-opt IL2 produced by cells responding to a 
stronger antigen to divide more times in co-culture settings and thus reduce the disparity with

2 cells responding the stronger antigen [52]. This was shown to be due to the growth effects of IL2 via PI3K signaling that triggers entry into cell cycle (Figure 1c). Additional critical nodes of integration can be gleaned from recently reported studies: 1) Notch1 is activated by T cell

5 signaling in cis and additional Notch1/2 expression is triggered by IL2 signaling [26, 53]. 2)

6 Further, TCR signaling induces digital expression of Myc, which is subsequently scaled by the

7 level of IL2 signaling [54]. Surprisingly, integration of TCR and IL2 signaling starts even within

8 the first 12 hours [52]. Thus, IL2 works to reduce heterogeneity from the earliest stages of T cell

9 activation and clonal expansion. This may be the case of additional cytokines produced by innate

10 cells such as IL12, IL1 $\beta$, IL6 and IFN $\alpha$ that are known to shape the early stages of T cell

11 proliferation and differentiation [55].

\section{Regulatory mechanisms facilitating a scaled response}

The detailed characterization of scaling in IL2 accumulation exemplifies that multiple regulatory mechanisms act in concert to enable a scaled response. We recognize and propose additional regulatory mechanisms that promote a scaled response based on: 1) parallels drawn from other signal-response systems; 2) accumulated functional, gene-expression and promoter/enhancer occupation data on transcription factors involved in T cell differentiation and clonal expansion, and 3) temporal gene-expression profile of TCR transgenic T cells responding to antigen in vivo. We provide additional credence to these regulatory mechanisms by highlighting theoretical and quantitative analyses that demonstrate their contribution to a scaled response.

We can draw on design principles learned from the study of receptor tyrosine kinase signaling that links signal integration to cell division in multiple cell types. Messenger RNA of immediate early genes (IEGs), such as Fos, Jun and Myc, are transcribed in the first wave of the response to growth factors like platelet derived growth factor. These have high content of AU-rich elements that target them to degradation. Thus, messages of IEGs do not accumulate appreciably. Accumulation of protein products of IEGs and cyclinD is contingent on prolonged Erk and Akt signaling downstream of growth factor signaling to prevent their degradation and initiate entry in to S-phase [56, 57]. This constitutes a "type I coherent feed-forward interaction with AND 
1 logic”, which acts as a 'persistence detector' and decodes the duration of signaling [58]. IEG and

2 cyclinD accumulation during the priming stage of $\mathrm{T}$ cell activation should also be mediated by

3 the same feed-forward interaction through prolonged signaling (Figure 2a). AP4, a transcription

4 factor induced by Myc, has also been shown to be dependent on continuous TCR and IL2R

5 signaling to prevent its degradation and enable continuation of metabolic and biosynthetic

6 reprogramming [59].

7 Acceleration in the secretion of IL2 has been shown to contribute to scaling of IL2 accumulation

8 according to antigen dose [7]. The authors used the phenomenology of an intermediary positive

9 feedback to model acceleration in the production of IL2. We propose that this positive feedback manifests due to the generic action of mTor and Myc. Myc leads to transcriptional amplification of all active promoters in B cells [60]. mTor also increases the activity of all three classes of RNA polymerases. Further, both Myc and mTor are master regulators of ribosome biogenesis. mTor is expected to be continuously active during T cell priming due to continuous PI3K signaling. Additional gradual increase in mTor activity is expected due to the accumulation of the transporters responsible for uptake of amino acids from the extracellular milieu. With Myc also accumulating due to continuous signaling (Figure 2a), there is a global non-linear increase in the transcriptional and translational output [60,61]. We posit that this phenomenon during the priming stage of $\mathrm{T}$ cell activation enables scaling of every aspect of the response and not just that of IL2.

Detailed examination of targets of master regulators involved in clonal expansion revealed to us several instances of two master regulators acting synergistically, with one of them also inducing the other master regulator (Figure 2b) (Supplementary Note 2). This type of interaction is called a "Type I coherent feed-forward with SUM logic" [62]. Such a regulatory interaction imparts two functional features: 1) accumulation of the end-product can accelerate over time and reach levels not possible due to a single transcription factor due to the SUM logic. 2) Transcription of the end-product can continue well after the initiating transcription factor activity has ceased [62]. The first feature contributes to scaling of the response. The second feature provides an explanation for how clonal expansion and differentiation can proceed well after the TCR signaling has ceased $[3,10,59]$. It is worth noting that the gleaned examples also include cases of cascading feed-forwards, which further pronounce the above described functional features. 
Inflammatory and stress response systems are characterized by digital signaling leading to graded transcriptional response [63-65]. These systems contain regulatory features that enable 'dose-to-duration' encoding [66]. If dose scales the duration of digital signaling, then temporal integration during transcription/translation is theoretically sufficient for scaling the response. Thus, 'dose-to-duration' encoding is arguably the most important feature of digital signaling systems. There is emerging evidence that $\mathrm{T}$ cells contain an analogous encoding mechanism to translate antigenic dose and strength to duration of signaling. Higher antigen dose and strength increase the net T-DC contact duration in vivo [67-69], which will automatically increase the duration of signaling and thus enable scaling by temporal integration. However, it is not known how T cells achieve this. We propose a mechanism for 'dose/strength-do-duration' encoding in the next section.

\section{Positive and negative feedbacks for scaling the response}

As part of the efforts of the Immgen Consortium, we have previously reported gene expression profiling of OT1 TCR transgenic T cells undergoing priming in vivo due to infection with ovalbumin expressing Listeria [12]. Functional classification of the transcripts with decreased expression during the first 48 hours revealed system-wide reduction in the transcripts for the motility apparatus and the TCR signaling machinery (Supplementary Figure S1-S4). Based on these system-wide changes we propose transcriptional positive and negative feedbacks and explain how they can contribute towards scaling the response.

Balance between the opposing 'stop' and 'go' signals determine the duration of contact with the antigen-presenting dendritic cells [70]. The current view based on collective evidence is that receptor proximal signalling constitutes the 'stop' signal in T cells. Continuous TCR triggering is known to reduce the receptor proximal signalling output due to activation induced degradation of tyrosine phosphorylated signalling proteins [71]. This is expected to gradually reduce the 'stop' signal until a lower steady state signaling is approached after $\sim 6$ hours. Unless there is a reduction in the 'go' signals as well, we anticipate that $\mathrm{T}$ cells will not be able to maintain and prolong the interaction with the engaged dendritic cells after the first $\sim 6$ hours. In fact, Jens Stein's lab has recently documented a T-cell intrinsic suppression of motility behavior in the first 2-3 days after antigen encounter in CD4 T cells [72]. We have found that the entire motility 
apparatus, encompassing chemokine receptors, integrins, modulators and effectors of Rho family

2 GTPases, adhesome components and actin binding proteins, had $~ 50 \%$ decrease in message levels during the priming stage (Supplementary Figure S1 and S2). We propose that system-wide suppression of both amoeboid and mesenchymal motility modes disables both the cell-intrinsic and extrinsic 'go'-signals and enables prolonged TCR signalling as a result of extended contact with engaged DCs. Since initial TCR signaling transcriptionally suppresses the motility apparatus, a slow positive-feedback is formed to increase the duration of TCR signaling (Figure

8 3a, Key Figure). If the extent of suppression in the motility apparatus is proportional with TCR 9 signaling strength, the observed phenomenon can cause the response to a stronger signal to widen further from a weaker signal. It is well-established, through studies of TNF $\alpha$ response and efforts to build analog synthetic circuits, that transcriptional positive feedback can extend the dynamic range of the response [73, 74]. Thus, we offer a mechanistic explanation for how 'dose/strength-to-duration' encoding occurs to scale the T cell response.

We have also observed $~ 50 \%$ reduction in the messages of nearly all TCR proximal signaling proteins as well as that of proteins constituting every major pathway of signaling downstream of TCR, including calcium, MAPK, NF-kB, Akt and mTor (Supplementary Figure S3 and S4). Over prolonged or continuous TCR signaling beyond $\sim 6$ hours, this phenomenon is likely to operate as a negative feedback, wherein the TCR signaling output is gradually reduced by a sizeable fraction (Figure 3b). A well-characterized transcriptional negative feedback is that of

Erk induced production of its phosphatases, which was also the first case of negative feedback to have been demonstrated to linearize the response [75]. This specific negative feedback is also expected to be operational during T cell priming. Multiple other natural and synthetic signal response systems are benefited by transcriptional negative feedbacks in increasing the dynamic range by linearizing the response (Supplementary Note 3). We anticipate that the signaldependent reduction in the TCR signaling output due to system-wide decrease in the expression of pathway components also contributes to linearizing and scaling the T cell response. How is the negative feedback on the TCR signaling apparatus enforced? We propose two parallel mechanisms (Figure 3b). It is known that several miRNAs are induced during the $\mathrm{T}$ cell priming stage [76]. Interestingly, signaling proteins were highly enriched among the predicted targets of 
the induced miRNAs. Thus, we propose that miRNAs expressed in response to TCR signaling target the entire TCR signaling apparatus. A second set of evidence supports this hypothesis. Dicer-deficient CD8 T cells, which don't have mature miRNAs, exhibit faster initial response and also produce more effector molecules [77, 78]. More importantly, Dicer-deficient CD4 T cells secrete IL2 without co-stimulatory signals and thus cannot distinguish between anergic and immunogenic signals [79]. In other words, absence of critical miRNAs reduces the dynamic range of the $\mathrm{T}$ cell response, which as per our hypothesis is due to the absence of the proposed negative feedback in Dicer-deficient T cells. A second mechanism may also contribute towards reducing the expression of TCR signaling proteins. Bcl11b and Tcf1 are important drivers of the T-cell lineage program and contribute to the expression of proteins that participate in TCR signaling [80]. We have found that priming of OT1 T cells leads to reduction in the levels of Bcl11b and Tcf1, which is expected to reduce the expression of TCR signaling proteins.

\section{Concluding remarks}

Scaling of the T cell response as per the antigenic dose and strength is crucial for an optimal immune response to resolve the infection with minimal damage to the host tissues. In this article we have attempted to build a unifying conceptual framework to explain scaling in T cell response. Scaled response is made possible by a ‘dose/strength-to-duration' encoding, wherein the duration of digital TCR signaling is scaled according to the antigenic dose and strength. Scaled accumulation of critical transcriptional intermediates as per the duration of digital TCR signaling, then programs the T cell response. Numerous feedforward interactions in the transcriptional program allow for decoding the duration of signaling, and for increasing and extending the response well beyond the cessation of signaling. The proposed feedback regulatory interactions in the transcriptional program improve the linearity of the response and extend the dynamic range of the $\mathrm{T}$ cell response. The shared nature of cytokine production and consumption is expected to reduce cellular heterogeneity to enable a coherent, collective $\mathrm{T}$ cell response.

\section{Acknowledgement}


MLD is supported by a Wellcome Trust Principal Research Fellowship and an ERC Advanced Grant (No. 670930). We apologise to authors whose work is not directly cited owing to space constraints.

\section{References}

1 Blander, J.M. and Sander, L.E. (2012) Beyond pattern recognition: five immune checkpoints for scaling the microbial threat. Nat Rev Immunol 12, 215-225

2 Zhang, N. and Bevan, Michael J. (2011) CD8+ T Cells: Foot Soldiers of the Immune System. Immunity 35, 161-168

3 Obst, R. (2015) The timing of T cell priming and cycling. Frontiers in Immunology 6, 563

4 van Heijst, J.W.J., et al. (2009) Recruitment of Antigen-Specific CD8+ T Cells in Response to Infection Is Markedly Efficient. Science 325, 1265-1269

5 Corse, E., et al. (2011) Strength of TCR-Peptide/MHC Interactions and In Vivo T Cell Responses. The Journal of Immunology 186, 5039-5045

6 Tscharke, D.C., et al. (2015) Sizing up the key determinants of the CD8+ T cell response. Nat Rev Immunol 15, 705-716

7 Tkach, K.E., et al. (2014) T cells translate individual, quantal activation into collective, analog cytokine responses via time-integrated feedbacks. eLife 3, e01944

8 Zehn, D., et al. (2009) Complete but curtailed T-cell response to very low-affinity antigen. Nature 458, 211-214

9 King, Carolyn G., et al. (2012) T Cell Affinity Regulates Asymmetric Division, Effector Cell Differentiation, and Tissue Pathology. Immunity 37, 709-720

10 Marchingo, J.M., et al. (2014) T cell signaling. Antigen affinity, costimulation, and cytokine inputs sum linearly to amplify T cell expansion. Science 346, 1123-1127

11 Enouz, S., et al. (2012) Autoreactive T cells bypass negative selection and respond to self-antigen stimulation during infection. The Journal of Experimental Medicine 209, 1769-1779

12 Best, J.A., et al. (2013) Transcriptional insights into the CD8+ T cell response to infection and memory T cell formation. Nat Immunol 14, 404-412

13 Cantrell, D. (2015) Signaling in Lymphocyte Activation. Cold Spring Harbor Perspectives in Biology 7 14 Tkach, K. and Altan-Bonnet, G. (2013) T cell responses to antigen: hasty proposals resolved through long engagements. Current Opinion in Immunology 25, 120-125

15 Jenkins, M.K. and Moon, J.J. (2012) The Role of Naive T Cell Precursor Frequency and Recruitment in Dictating Immune Response Magnitude. The Journal of Immunology 188, 4135-4140

16 Lanzavecchia, A. and Sallusto, F. (2001) Antigen decoding by T lymphocytes: from synapses to fate determination. Nat Immunol 2, 487-492

17 Finetti, F., et al. (2014) Specific recycling receptors are targeted to the immune synapse by the intraflagellar transport system. Journal of Cell Science 127, 1924-1937

18 Willinger, T., et al. (2015) Dynamin 2-dependent endocytosis sustains T-cell receptor signaling and drives metabolic reprogramming in T lymphocytes. Proceedings of the National Academy of Sciences $112,4423-4428$ 
19 Becker, V., et al. (2010) Covering a Broad Dynamic Range: Information Processing at the Erythropoietin Receptor. Science 328, 1404-1408

20 Das, J., et al. (2009) Digital Signaling and Hysteresis Characterize Ras Activation in Lymphoid Cells. Cell 136, 337-351

21 Celli, S., et al. (2005) CD4 T cells integrate signals delivered during successive DC encounters in vivo. The Journal of Experimental Medicine 202, 1271-1278

22 Clark, C.E., et al. (2011) A Role for the Immediate Early Gene Product c-fos in Imprinting T Cells with Short-Term Memory for Signal Summation. PLOS ONE 6, e18916 23 Marangoni, F., et al. (2013) The Transcription Factor NFAT Exhibits Signal Memory during Serial T Cell Interactions with Antigen-Presenting Cells. Immunity 38, 237-249

24 Rosette, C., et al. (2001) The Impact of Duration versus Extent of TCR Occupancy on T Cell Activation: A Revision of the Kinetic Proofreading Model. Immunity 15, 59-70

25 Holst, J., et al. (2008) Scalable signaling mediated by T cell antigen receptor-CD3 ITAMs ensures effective negative selection and prevents autoimmunity. Nat Immuno/ 9, 658-666

26 Guy, C.S., et al. (2013) Distinct TCR signaling pathways drive proliferation and cytokine production in T cells. Nat Immunol 14, 262-270

27 Dang, C.V. (2013) MYC, metabolism, cell growth, and tumorigenesis. Cold Spring Harbor perspectives in medicine 3

$28 \mathrm{Nie}, \mathrm{Z}$., et al. (2012) c-Myc is a universal amplifier of expressed genes in lymphocytes and embryonic stem cells. Cell 151, 68-79

29 Wang, R., et al. (2011) The transcription factor Myc controls metabolic reprogramming upon T lymphocyte activation. Immunity $35,871-882$

30 Palomero, T., et al. (2006) NOTCH1 directly regulates c-MYC and activates a feed-forward-loop transcriptional network promoting leukemic cell growth. Proceedings of the National Academy of Sciences of the United States of America 103, 18261-18266

31 Backer, R.A., et al. (2014) A central role for Notch in effector CD8+ T cell differentiation. Nature Immunology 15, 1143-1151

32 Kinjyo, I., et al. (2015) Real-time tracking of cell cycle progression during CD8+ effector and memory T-cell differentiation. Nature communications 6, 6301

33 Man, K., et al. (2013) The transcription factor IRF4 is essential for TCR affinity-mediated metabolic programming and clonal expansion of T cells. Nat Immunol 14, 1155-1165

34 Nayar, R., et al. (2014) Graded levels of IRF4 regulate CD8+ T cell differentiation and expansion, but not attrition, in response to acute virus infection. Journal of immunology (Baltimore, Md. : 1950) 192, 5881-5893

35 Kurachi, M., et al. (2014) The transcription factor BATF operates as an essential differentiation checkpoint in early effector CD8+ T cells. Nat Immunol 15, 373-383

36 Godec, J., et al. (2015) Inducible RNAi in vivo reveals that the transcription factor BATF is required to initiate but not maintain CD8+ T-cell effector differentiation. Proceedings of the National Academy of Sciences of the United States of America 112, 512-517

37 Yao, S., et al. (2013) Interferon regulatory factor 4 sustains CD8(+) T cell expansion and effector differentiation. Immunity 39, 833-845

38 Kaern, M., et al. (2005) Stochasticity in gene expression: from theories to phenotypes. Nat Rev Genet 6, 451-464

4439 Buchholz, V.R., et al. (2014) T Cell Fate at the Single-Cell Level. Annual Review of Immunology

4540 Duffy, K.R. and Hodgkin, P.D. (2012) Intracellular competition for fates in the immune system. Trends

46 in Cell Biology 22, 457-464

4741 Murphy, K.M. and Stockinger, B. (2010) Effector T cell plasticity: flexibility in the face of changing

48 circumstances. Nat Immunol 11, 674-680 
42 Plumlee, C.R., et al. (2015) Early Effector CD8 T Cells Display Plasticity in Populating the Short-Lived Effector and Memory-Precursor Pools Following Bacterial or Viral Infection. Scientific Reports 5, 12264 43 Handly, L.N., et al. (2015) Paracrine communication maximizes cellular response fidelity in wound signaling. eLife 4, e09652

44 Waysbort, N., et al. (2013) Coupled IL-2-dependent extracellular feedbacks govern two distinct consecutive phases of CD4 T cell activation. Journal of immunology (Baltimore, Md. : 1950) 191, 58225830

45 Busse, D., et al. (2010) Competing feedback loops shape IL-2 signaling between helper and regulatory T lymphocytes in cellular microenvironments. Proceedings of the National Academy of Sciences of the United States of America 107, 3058-3063

46 Chung, J., et al. (1997) STAT3 serine phosphorylation by ERK-dependent and -independent pathways negatively modulates its tyrosine phosphorylation. Molecular and cellular biology 17, 6508-6516 47 Vanhatupa, S., et al. (2008) MAPK-induced Ser727 phosphorylation promotes SUMOylation of STAT1. The Biochemical journal 409, 179-185

48 Verdeil, G., et al. (2006) STAT5-mediated signals sustain a TCR-initiated gene expression program toward differentiation of CD8 T cell effectors. Journal of immunology (Baltimore, Md. : 1950) 176, 48344842

49 Wang, C.J., et al. (2012) Diverse sensitivity thresholds in dynamic signaling responses by social amoebae. Sci Signal 5, ra17

50 Xue, Q., et al. (2015) Analysis of single-cell cytokine secretion reveals a role for paracrine signaling in coordinating macrophage responses to TLR4 stimulation. Sci Signal 8, ra59

51 Sabatos, C.A., et al. (2008) A synaptic basis for paracrine interleukin-2 signaling during homotypic $T$ cell interaction. Immunity 29, 238-248

52 Voisinne, G., et al. (2015) T Cells Integrate Local and Global Cues to Discriminate between Structurally Similar Antigens. Cell reports 11, 1208-1219

53 Mathieu, M., et al. (2015) The Notch signaling pathway controls short-lived effector CD8+ T cell

differentiation but is dispensable for memory generation. Journal of immunology (Baltimore, Md. : 1950) 194, 5654-5662

54 Preston, G.C., et al. (2015) Single cell tuning of Myc expression by antigen receptor signal strength and interleukin-2 in T lymphocytes. The EMBO journal 34, 2008-2024

55 Starbeck-Miller, G.R., et al. (2014) IL-12 and type I interferon prolong the division of activated CD8 T cells by maintaining high-affinity IL-2 signaling in vivo. J Exp Med 211, 105-120

56 Murphy, L.O., et al. (2004) A network of immediate early gene products propagates subtle differences in mitogen-activated protein kinase signal amplitude and duration. Molecular and cellular biology 24, 144-153

57 Torii, S., et al. (2006) ERK MAP kinase in G cell cycle progression and cancer. Cancer science 97, 697702

58 Mangan, S. and Alon, U. (2003) Structure and function of the feed-forward loop network motif. Proceedings of the National Academy of Sciences of the United States of America 100, 11980-11985 59 Chou, C., et al. (2014) c-Myc-induced transcription factor AP4 is required for host protection mediated by CD8+ T cells. Nat Immunol 15, 884-893

60 Wolf, E., et al. (2015) Taming of the beast: shaping Myc-dependent amplification. Trends Cell Biol 25, 241-248

4461 Levens, D. (2013) Cellular MYCro economics: Balancing MYC function with MYC expression. Cold 45 Spring Harbor perspectives in medicine 3

4662 Kalir, S., et al. (2005) A coherent feed-forward loop with a SUM input function prolongs flagella

47 expression in Escherichia coli. Molecular systems biology 1, 2005.0006 
63 English, J.G., et al. (2015) MAPK feedback encodes a switch and timer for tunable stress adaptation in yeast. Sci Signal 8, ra5

64 Tay, S., et al. (2010) Single-cell NF-kappaB dynamics reveal digital activation and analogue information processing. Nature 466, 267-271

65 Lopez, J.M. (2010) Digital kinases: A cell model for sensing, integrating and making choices.

Communicative \& integrative biology 3, 146-150

66 Behar, M., et al. (2008) Dose-to-duration encoding and signaling beyond saturation in intracellular signaling networks. PLoS computational biology 4, e1000197

67 Henrickson, S.E., et al. (2013) Antigen availability determines CD8(+) T cell-dendritic cell interaction kinetics and memory fate decisions. Immunity 39, 496-507

68 Moreau, H.D., et al. (2012) Dynamic in situ cytometry uncovers T cell receptor signaling during immunological synapses and kinapses in vivo. Immunity 37, 351-363

69 van Panhuys, N., et al. (2014) T-cell-receptor-dependent signal intensity dominantly controls CD4(+) T cell polarization In Vivo. Immunity 41, 63-74

70 Dustin, M.L. (2004) Stop and go traffic to tune T cell responses. Immunity 21, 305-314

71 Jang, I.K. and Gu, H. (2003) Negative regulation of TCR signaling and T-cell activation by selective protein degradation. Curr Opin Immunol 15, 315-320

72 Ackerknecht, M., et al. (2015) In vivo TCR Signaling in CD4(+) T Cells Imprints a Cell-Intrinsic, Transient Low-Motility Pattern Independent of Chemokine Receptor Expression Levels, or Microtubular Network, Integrin, and Protein Kinase C Activity. Front Immunol 6, 297

73 Daniel, R., et al. (2013) Synthetic analog computation in living cells. Nature 497, 619-623

74 Sung, M.H., et al. (2014) Switching of the relative dominance between feedback mechanisms in lipopolysaccharide-induced NF-kappaB signaling. Sci Signal 7, ra6

75 Bhalla, U.S., et al. (2002) MAP kinase phosphatase as a locus of flexibility in a mitogen-activated protein kinase signaling network. Science 297, 1018-1023

76 Grigoryev, Y.A., et al. (2011) MicroRNA regulation of molecular networks mapped by global microRNA, mRNA, and protein expression in activated T lymphocytes. Journal of immunology (Baltimore, Md. : 1950) 187, 2233-2243

77 Trifari, S., et al. (2013) MicroRNA-directed program of cytotoxic CD8+ T-cell differentiation. Proceedings of the National Academy of Sciences of the United States of America 110, 18608-18613 78 Zhang, N. and Bevan, M.J. (2010) Dicer controls CD8+ T-cell activation, migration, and survival. Proceedings of the National Academy of Sciences of the United States of America 107, 21629-21634 79 Marcais, A., et al. (2014) microRNA-mediated regulation of mTOR complex components facilitates discrimination between activation and anergy in CD4 T cells. J Exp Med 211, 2281-2295 80 Yui, M.A. and Rothenberg, E.V. (2014) Developmental gene networks: a triathlon on the course to T cell identity. Nat Rev Immunol 14, 529-545

\section{Figure legends:}

Figure 1: Regulatory interactions between TCR and IL2R signaling for a collective, coherent, scaled response. a) TCR signaling leads to IL2 and IL2R transcription. IL2 accumulation occurs due to suppression of Stat5 phosphorylation by TCR signaling and because of a putative positive feedback. Beyond 48 hours, IL2R signaling leads to further IL2R transcription, but suppression 
of IL2 transcription. Further, IL2 is degraded after internalization. b) Profiles of IL2 and IL2R as

2 dictated by the regulatory interactions. c) PI3K, Notch, and Myc respond to both TCR and IL2R

3 signaling, acting as nodes of integration for a coherent growth program in responding cells. Note

4 that the effect of mTOR on Myc and cell growth and cell-cycle machinery is not just by

5 transcriptional means.

6

7 Figure 2: Coherent feed forward interactions promote a scaled T cells response. a) Erk and Akt 8 signaling downstream of TCR and IL2R signaling ensure accumulation of the protein products 9 by preventing their degradation for the duration of signaling. This feed-forward interaction with AND logic is highly sensitive to loss of upstream signaling, leading to sudden drop in the output.

11 Accumulation of the secondary products, cyclin D and AP4, is even more sensitive to the 12 duration of signaling due to the cascading induction. b) Individual examples of feed-forward 13 interactions with SUM logic in the transcriptional programs during T cell activation. These were 14 inferred based on the collective knowledge on these transcription factors, especially in the context of T cell activation (Supplementary Note 2). These increase the dynamic range of the response due to continuous increase in the rate of production of the end-product and also extend

17 the response well after the cessation of TCR signaling.

Figure 3: Proposed positive and negative feedbacks in scaling the T cell response. a) Contact duration with DCs is known to be influenced by the dose and strength of antigen presented by the DCs. The initial arrest is due to the TCR mediated 'stop' signal, represented as the 'fast'

22 double negative feedback. However, over time the TCR signal diminishes and the stop signal is reinforced by a slow transcriptional feedback that reduces the expression of the entire motility apparatus as per the signaling output. This transcriptional positive feedback increases the duration of signaling and enables scaling of the response. b) TCR signaling also leads to

26 reduction in the messages of the signaling machinery. This negative feedback is enforced via

27 microRNAs and lineage determining factors Bcl11b and Tcf1. Transcriptional negative feedback 28 is known to linearlize the response. 